
Pathophysiology of Haemostasis and Thrombosis

Received: March 23, 2001

Accepted in revised form: April 22, 2002

\title{
Thromboplastin Bilbao: Reproducibility and Sensitivity of a Spanish Thromboplastin
}

\author{
M. Vacasa M.J. Aguirrebeitiab P.J. Lafuente ${ }^{a, c}$ I. Unanue ${ }^{a}$ J .A. Iriarte ${ }^{a, b}$ \\ aFundación para la Investigación y Docencia de las Enfermedades Cardiovasculares, bServicio de Hematología, \\ Hospital de Basurto, 'Departamento de Medicina Preventiva y Salud Pública, Universidad del País Vasco/Euskal \\ Herriko Unibertsitatea, Bilbao, Spain
}

\author{
Key Words \\ Thromboplastin Bilbao • Prothrombin time . \\ Reproducibility assays
}

\begin{abstract}
Prothrombin time (PT) is the control test for oral anticoagulant therapy as well as the screening test for defects of the extrinsic pathway of coagulation. Its responsiveness to decreased extrinsic clotting factors depends on the source and type of tissue factor thromboplastin extract. In 1994, a rabbit brain thromboplastin - Thromboplastin Bilbao (TBi) - was introduced as a replacement for a human brain preparation used since 1983, with the aim of establishing a national standard. The purpose of this study was to check the reproducibility, the interassay/intra-assay accuracy and the stability of this reagent under temperature changes and over time. A method modified from Frei et al. [World Health Organisation Regional Publications, Eastern Mediterranean Series, Alexandria, 1995] was used for the preparation of thromboplastin extract. Thirty-five batches of human TBi were prepared from 1983 to 1988, while from 1993 to 199913 batches of rabbit TBi were produced. The interassay reproducibility of rabbit TBi exhibited a coefficient of variation (CV) of 1.07-1.57\% for normal plasma and of 1.25-2.56\% for anticoagulated plasma. The intra-assay CV was $0.06-1.30 \%$ for normal plasma and $1.23-2.66 \%$
\end{abstract}

for anticoagulated plasma. The stability of the reagent to temperature changes and time was also estimated, with similar results for the two thromboplastins. As a result of the Oral Anticoagulant Treatment Quality Assessment Scheme in the Basque Country, an in-house rabbit thromboplastin with good sensitivity and reproducibility was developed.

Copyright $\odot$ 2002 S. Karger AG, Basel

\section{Introduction}

Thromboplastins are reagents containing tissue factor and coagulant phospholipids [1] which can be obtained from a number of human and animal tissues. They vary in sensitivity to coagulation defects, with human extracts being more sensitive to decreased factors II, VII and X than those derived from animal tissues [2-4]. Some commercial preparations are particularly insensitive to factor VII, which is not surprising given the difficulty in avoiding blood vessel 'tissue contamination' when obtaining the extract and the complex chemical process required to eliminate the contamination. The loss of sensitivity to factor VII could have serious consequences during the early treatment stage, when only this factor is affected.

Another, perhaps primary, reason for the reduced sensitivity of certain reagents is the 'species-specific' phenomenon [5].

\begin{tabular}{ll}
\hline KARGER & (c) 2002 S. Karger AG, Basel \\
1424-8832/02/0323-0115\$18.50/0 \\
$\begin{array}{l}\text { Fax +4161306 1234 } \\
\text { www.karger.com }\end{array}$ & $\begin{array}{l}\text { Accessible online at: } \\
\text { www.karger.com/journals/pht }\end{array}$
\end{tabular}

Dr. Marta Vacas
c/ Ribera, 23-4
E-48005 Bilbao (Spain)
Tel. +34 944158765 , E-Mail bdbvarim@lg.ehu.es 
Oral anticoagulant drugs derived from coumarin are widely used in the treatment and prophylaxis of thrombotic disorders. The drug dosage must be adjusted periodically to achieve an adequate degree of anticoagulation. To overcome the problem of variability in the response of thromboplastins, the World Health Organization (WHO) introduced a calibration system in 1983 using reference thromboplastins as the basis for a standardized technique. It also introduced a key term for developing a standard way to express results - the International Normalized Ratio (INR). The introduction of INR has not only provided a common scale for oral anticoagulation, but has also facilitated recommendations for optimal therapeutic ranges of INR [5].

A sensitive thromboplastin extract has the advantage of allowing longer coagulation times and therefore a broader therapeutic range. Less sensitive reagents allow shorter times and, therefore, narrower therapeutic ranges, making it more difficult for the clinician to establish reliable doses. The qualities required for a good thromboplastin reagent can be summarized as: precise sensitivity to the effects produced by coumarins and by variations in factor VII, stability, reproducibility, good end point and easy, uniform dissolution.

From that moment on, specialists interested in standardizing oral anticoagulant treatments focused on promoting the development of high-sensitivity thromboplastins (ISI close to 1.0) and international use of INR. As a result, National Standardization Committees began setting up recommendations in this regard for publication in their respective countries. In Spain, numerous studies have been conducted since the early 1970 s to detect variations in PT technique and then develop a standard thromboplastin reagent against which all other reagents could be tested [7-9].

Given this background, we initiated a line of research in 1983 to produce a thromboplastin reagent called Thromboplastin Bilbao (TBi), and to calibrate it against international standard preparations so that it could be used as a standard reagent both in the Basque Country and in the rest of Spain.

\section{Material and Methods}

Production of the Thromboplastin Reagent

A human brain tissue extract was prepared until 1989, when it was replaced by a rabbit brain extract due to the risks entailed in the use of human thromboplastins. In both cases, the reagents were prepared using the tissue factor saline extract technique developed by Poller [10] and modified by Frei et al. [11].

\section{Prothrombin Time}

The manual technique (tilt tube) was used to perform the test in all phases of the study [12]. The methodology was as follows:

Warm test tubes to perform the test in a $37^{\circ} \mathrm{C}$ water bath. Resuspend the thromboplastin by gentle inversion and using an accurate manual or automatic $0.1-\mathrm{ml}$ pipette. Transfer $0.1 \mathrm{ml}$ into a glass test tube in the water bath. Allow to warm for 1-2 min. Thromboplastin should not be allowed to prewarm in the test tubes for more than $1 \mathrm{~h}$. Add $0.1 \mathrm{ml}$ plasma and after approximately $1 \mathrm{~min}$ add $0.1 \mathrm{ml}$ prewarmed $\left(37^{\circ} \mathrm{C}\right)$ calcium chloride $(0.025 \mathrm{~mol} / \mathrm{l})$ and start the stopwatch. Tilt gently, keeping the tube under water as much as possible to maintain the temperature. The speed and angle of tilting the test tube must be standardized to control glass activation and minimize cooling (three tilts through $90^{\circ}$ every $5 \mathrm{~s}$ is recommended). The end point, i.e. the appearance of the fibrin clot, is recorded.

Tests were performed in duplicate.

\section{Assessment of the Accuracy and Reproducibility of the Reagent}

The laboratory procedure for evaluating the accuracy of TBi involved measuring both intra-assay and inter-assay variations. To this end, the mean, the standard deviation and the coefficient of variation $(\mathrm{CV})$ were calculated. Two different plasma pools were employed: a pool of 20 normal plasma and a pool of plasma from 20 anticoagulated patients with INR levels within the therapeutic range (2.0-4.0).

Intra-Assay Variation. Determined daily for 10 days performing PT in triplicate in both pools five times. The PT test results were recorded in seconds and the $\mathrm{CV}$ was calculated each day from the mean PT for each run. The CV reported was calculated from the 5 mean values.

Inter-Assay Variation. The same pools were frozen at $-20^{\circ} \mathrm{C}$ and analyzed in triplicate for 10 consecutive days. PT results were also evaluated in seconds.

\section{Stability}

The batches were evaluated both for stability over time and for stability to temperature changes. All the tests were performed in parallel with the Manchester Reagent thromboplastin (National Laboratory for Anticoagulant Reagents and Control of the Withington Hospital, Manchester), the reagent used as routine reference material.

Stability Over Time. The TBi reagent is prepared in liquid form. Two freeze-dried plasma samples were used - a normal and a vitamin-K-deficient sample (Inmuno Diagnóstica) to evaluate the stability of TBi batches. One batch of TBi stored at $2-8^{\circ} \mathrm{C}$ was analyzed per month, measuring PT in duplicate in the two plasma samples for a period of one year. The test results were evaluated calculating the mean and standard deviation of the data obtained in seconds. The criterion for stability is based on the fact that a lengthening in the PT of normal plasma should not exceed two standard deviations compared to the mean normal value.

Effects of Temperature. An accelerated degradation test was performed [13]. We used a batch of thromboplastin and a pool of 20 fresh normal plasma samples plus another pool of stabilized anticoagulated patients. In both pools, PT was measured with the reagent incubated at 37 and $45^{\circ} \mathrm{C}$ and at different intervals of time (1-2 and 3-4 h). The results were expressed as means and standard deviations of the PT values in seconds. The criterion of stability was that the initial change in the prothrombin time of normal plasma should not exceed $0.05 \%$ per day. This initial change was calculated using only the first two measurements ( 1 and $2 \mathrm{~h}$ ). 


\section{Calibration Study}

We followed the calibration methodology proposed by the WHO [1]. The calibration procedure entails the determination of a series of prothrombin times, using normal and abnormal plasma samples or whole blood samples, with both the reference and the test thromboplastin. The tests are performed using either fresh samples from individual subjects (procedure 1) or freeze-dried or frozen plasma samples (procedure 2). Abnormal plasma samples for procedure 1 are obtained from patients undergoing long-term oral anticoagulant treatment. Freeze-dried or frozen plasma samples for procedure 2 may be pooled.

We calibrated the human TBi against the second International Reference Preparation of Thromboplastin, Human, Plain, coded $\mathrm{BCT} / 253$ and the rabbit TBi with the Third International Reference Reagent of Thromboplastin, Rabbit, Plain, and coded RBT/90.

All plasma samples used for this procedure complied with the WHO requirements for the Collection, Processing and Quality Control of Blood, Blood Components and Plasma Derivates [14].

Two procedures were used: calibration of a secondary standard using individual fresh plasma or blood samples (procedure 1) and calibration of individual batches of thromboplastin (procedure 2).

Procedure 1: Calibration of a Secondary Standard Using Individual Fresh Plasma or Blood Samples. Recommended for the calibration of secondary standards against the appropriate international reference preparation. This procedure consists of a set of tests using 20 fresh normal plasma samples and 60 fresh anticoagulated samples. It is recommended to select samples with INR values in the range 1.54.5. The procedure should be repeated on at least five separate occasions using fresh reagents on each occasion.

Procedure 2: Calibration of Individual Batches of Thromboplastin. This procedure may be carried out with 1 pooled normal plasma sample and 2 different pooled coumarin plasma samples or plasma samples artificially depleted of vitamin-K-dependent coagulation factors.

Statistical Evaluation. An orthogonal regression line shall be calculated on the basis of $\log$ PT of the plasma. log PT for the working reference thromboplastin system is plotted on the vertical axis and $\log$ PT for the test batch of thromboplastin on the horizontal axis. We calculate the slope (b), the International Sensitivity Index (ISI $\left.{ }_{w}\right)$, the standard error of the ISI $\left(\mathrm{s}_{\mathrm{b}}\right)$ and the coefficient of variation (\%) of the ISI of the working preparation, following these formulas:

$$
I S I_{w}=I S I_{I R P} \cdot b
$$

being ISI $_{\text {IRP }}$ the ISI of the reference thromboplastin

$$
C V(\%)=100 \cdot S_{b} / b \text { being } \mathrm{s}_{\mathrm{b}} \text { the standard error of } \mathrm{b}
$$

\section{Results}

\section{Production of TBi Reagent}

From 1984 to 1987, a total of 35 batches of human thromboplastin was produced. In 1989, human brainderived TBi stopped being made and was replaced by rabbit brain material. Following a period of tests and adaptation to the new conditions, batches of TBi began to be produced in series. A total of 13 batches was produced during the years between 1994 and 1999.

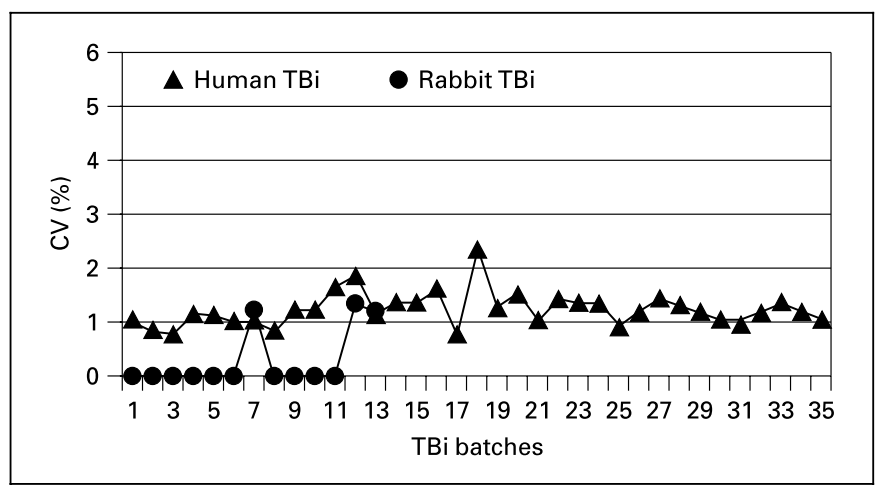

Fig. 1. Intra-assay reproducibility of human and rabbit TBi in normal plasma.

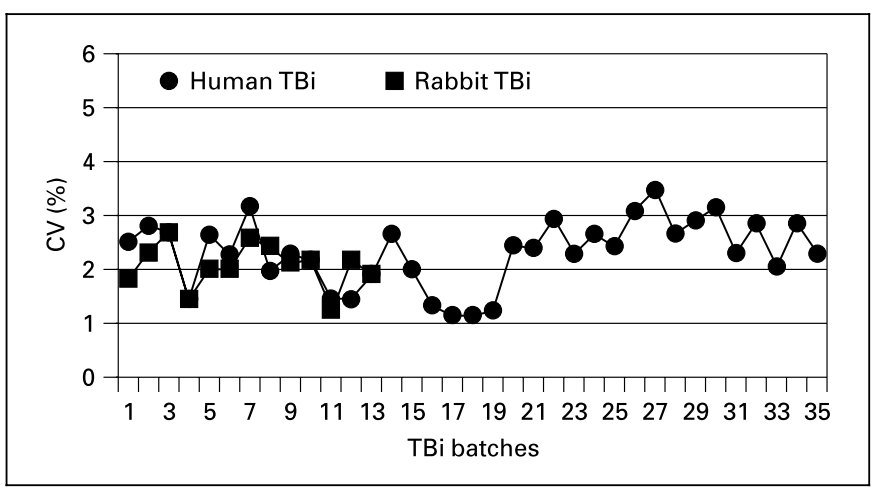

Fig. 2. Intra-assay reproducibility of human and rabbit TBi in anticoagulated plasma.

Methods of Assessing the Accuracy and Reproducibility of the Reagent: Intra-Assay and Inter-Assay Variation

Intra-Assay Reproducibility Study. Figure 1 shows the intra-assay accuracy for normal plasma with thromboplastins of both biological origins, while figure 2 shows the same evaluation for anticoagulated plasmas, also using both thromboplastins. The variation using human TBi ranged between 0.73 and $2.30 \%$ for the normal pool (PT interval $=11.56-13.13 \mathrm{~s}$ ), and between 1.11 and $3.45 \%$ for the pool of anticoagulated plasma (PT interval $=$ 30.00-46.91 s). For rabbit TBi, the normal pool had a coefficient of variation ranging between 0.06 and $1.30 \%$ $(\mathrm{PT}$ interval $=14.23-15.68 \mathrm{~s})$, while the anticoagulated plasma yielded a coefficient of variation ranging between 1.23 and $2.66 \%(\mathrm{PT}$ interval $=35.69-45.69 \mathrm{~s})$. 


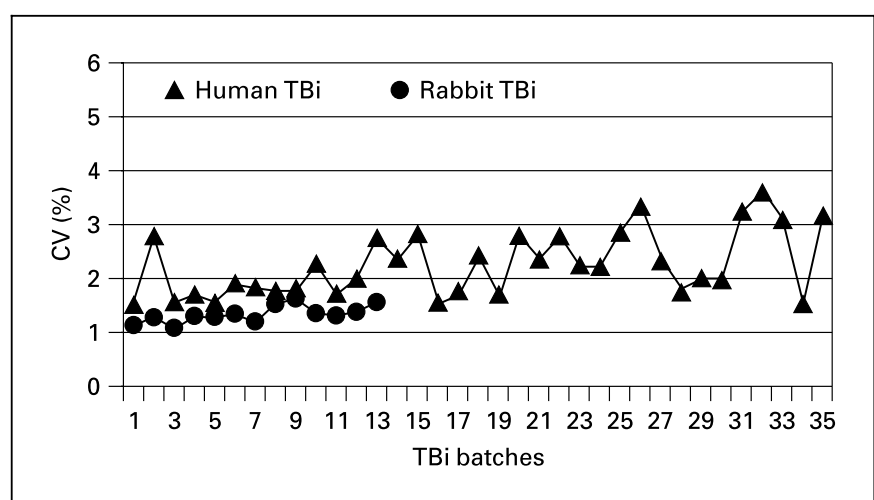

Fig. 3. Inter-assay reproducibility of human and rabbit TBi in normal plasma.

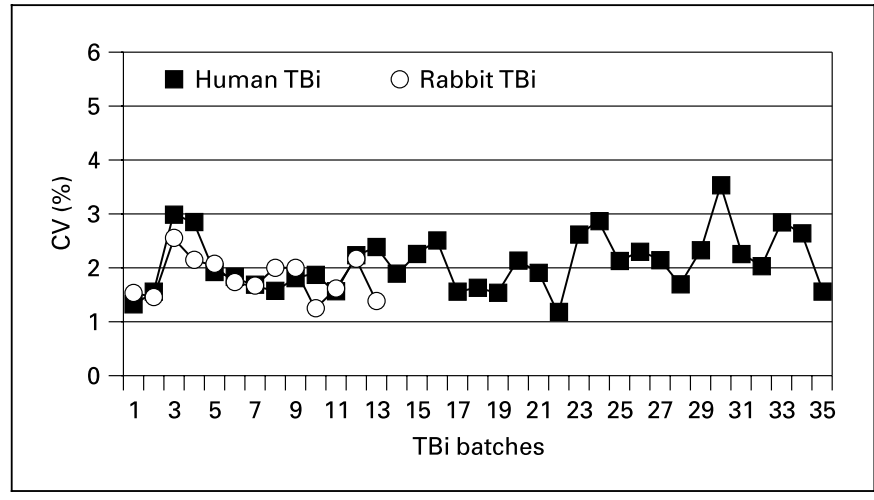

Fig. 4. Inter-assay reproducibility of human and rabbit TBi in anticoagulated plasma.

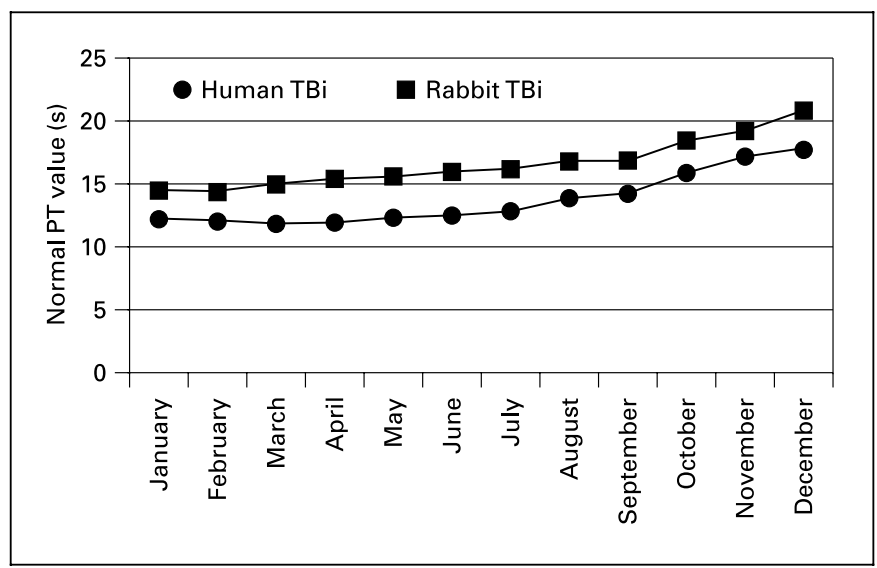

Fig. 5. Stability of TBi over time.
Inter-Assay Reproducibility Study. Figure 3 shows the coefficients of variation obtained by the two TBi reagents on analyzing the normal plasma pool, while figure 4 gives the results of the accuracy evaluation using anticoagulated plasma.

For human $\mathrm{TBi}$, the normality value presented a coefficient of variation ranging between 1.52 and $3.54 \%$ (PT interval $=11.7-13.18)$, while for the pool of anticoagulated plasma the range was between 1.18 and $3.25 \%$ (PT interval $=31.15-45.20$ ).

In the case of rabbit TBi batches, the coefficients of variation for the precision of normal plasma ranged between 1.07 and 1.57\% (PT interval $=14.70-16.00)$, and between 1.25 and $2.56 \%$ for the anticoagulated plasma pool $(\mathrm{PT}$ interval $=35.70-51.70)$.

\section{Stability Assessment of the Batches}

Stability over Time. Evaluations were made of the stability of rabbit- and human-derived batches over a period of one year. Figure 5 provides a comparison of the lengthening of normal PT values for TBi, taken as our criteria for evaluating stability over a period of 12 months (human TBi batch 21/85 and rabbit TBi batch 5/96). In both types of thromboplastins, stability was estimated at 5-6 months from the beginning of batch production.

Temperature Stability. Figure 6 shows that the response of thromboplastin is significantly altered if kept at $37^{\circ} \mathrm{C}$ for more than $6 \mathrm{~h}$, and that destabilization occurs much more quickly (in $4 \mathrm{~h}$ ) if the reagent is kept at a temperature of $45^{\circ} \mathrm{C}$.

\section{Calibration Study}

The ISI values of the 35 human TBi batches are presented in figure 7 . For this type of reagent, the ISI ranged between 0.99 and 1.15. The CV of ISI was less than $3 \%$ $(0.14-2.70 \%)$. For rabbit TBi, the ISI values of the 13 batches prepared ranged between 0.99 and 1.23 (also represented in fig. 7). The imprecision of ISI was also less than 3\% (0.94-1.81\%).

\section{Discussion}

In analyzing the production of thromboplastin reagent, the results were considered separately according to origin (rabbit brain or human brain). However, as the production technique remained the same in both phases, it is possible to compare the data obtained by reproducibility and sensitivity tests without taking into account any new variables. 
Fig. 6. Temperature stability of TBi.

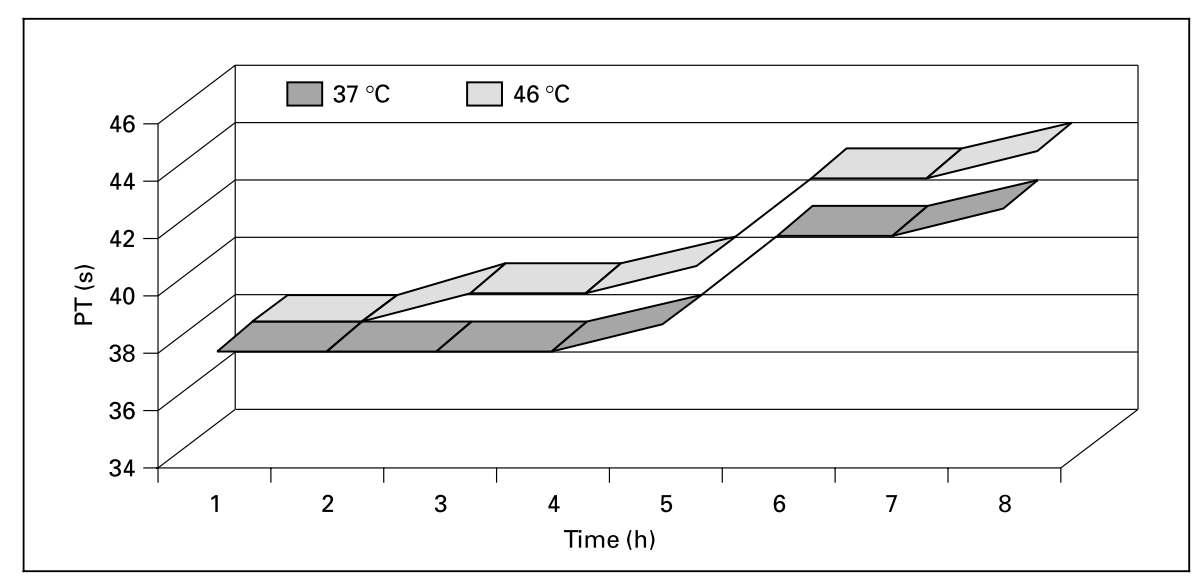

One of the most important requirements for a thromboplastin reagent is that variability be kept to a minimum in the batches produced. In the case of TBi, it was found that the same test (or intra-assay) variation in normal plasma ranged between 0.73 and $2.30 \%$ for the humanderived reagent, and between 0.06 and $1.30 \%$ for the rabbit-derived reagent, while for anticoagulated plasma, the values ranged between 1.11 and $3.45 \%$ for human and 1.23 and $2.66 \%$ for rabbit thromboplastin. These data do not differ from those reported in studies undertaken with international standard human, rabbit and, most recently, recombinant thromboplastins [15-17]. It should be noted that the variation was slightly less in the rabbit $\mathrm{TBi}$, particularly in the case of normal plasmas. This greater homogeneity could be due to better preservation of the plasma analyzed.

Regarding the inter-assay variations recorded for normal plasmas, the values found for both thromboplastins (1.52-3.54\% for human TBi and $1.07-1.57 \%$ for rabbit $\mathrm{TBi}$ ) show once again that there is less variation in the latter, and that the difference is more significant than in the previous case. Possibly this is due to the same reason, although both variation ranges are within those obtained using 'high-sensitivity' reagents [15-17]. In the case of anticoagulated plasma, the same situation arises (1.18$3.25 \%$ of variation for human thromboplastin and $1.25-$ $2.56 \%$ for rabbit $\mathrm{TBi}$ ).

One of the advantages of the 'sensitive' thromboplastin reagents is that their therapeutic ranges provide a wide margin in seconds. Certain low-sensitivity reagents allow an interval of only a few seconds between an anticoagulation level that would be therapeutically ineffective and a level that could be considered excessive, placing the patient at risk of hemorrhage. In many human-derived

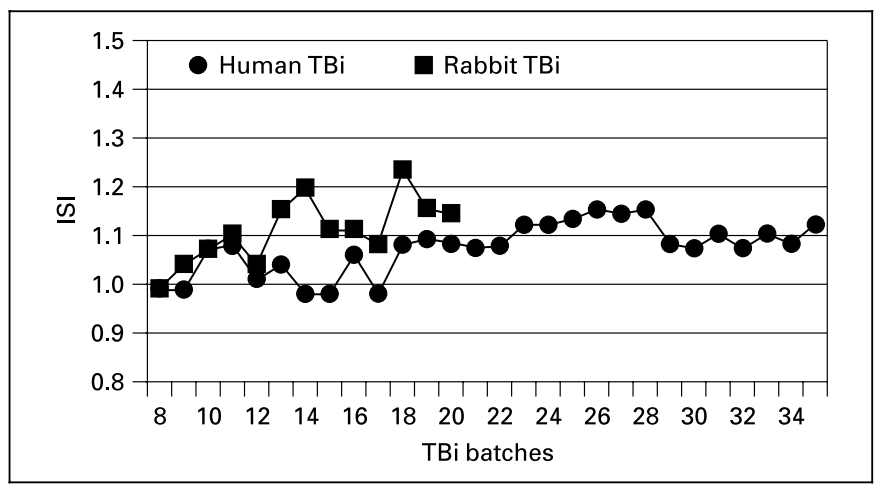

Fig. 7. ISI values of human and rabbit TBi.

thromboplastins, there is a margin of about $15 \mathrm{~s}$ in the therapeutic range, making it easier and safer for the clinician to adjust the dosage as needed, thus helping to prevent rethrombosis or thrombosis. In our case, human TBi had an INR therapeutic range of between 2 and 4 and a PT margin ranging between 24 and $47 \mathrm{~s}$, corresponding to a prothrombin activity time of between 10 and $25 \%$. This interval of almost $20 \mathrm{~s}$ concurs with the values reported for the British Comparative Reagent, which has the same source, preparation methodology and origin as one of the International Standard Preparations of the WHO [18, 19].

Regarding rabbit $\mathrm{TBi}$, and considering the same INR therapeutic range of between 2 and 4 - the range recommended in the 1980s for comparison with human reagents [20] - we found a PT range of between 29 and $54 \mathrm{~s}$, which is similar to the range reported for the Manchester Reagent produced in the United Kingdom and consid- 
ered the standard reagent for this study. These ranges have varied [21-23], with the maximum recommended range being 2-3.5 for heart valve prostheses, which in the case of our reagent would mean a PT of 29-47 s, a margin which is still too narrow to guarantee safe anticoagulation [24].

With regard to TBi stability, deterioration of the liquid reagent kept since production at $4{ }^{\circ} \mathrm{C}$ occurs at around 6 months. This is true for both human and rabbit thromboplastin. Compared to the British Comparative Thromboplastin and to the Manchester Reagent, both of which are produced using the same technique, expiry times are very similar $[24,25]$. This deterioration is attributed to the fact that production of the reagent calls for maceration of the brain, whether human or rabbit, causing rupture of the cell membranes and release of potentially oxidizing materials originating in subcellular particles. The loss of procoagulant activity is attributed to a loss of phospholipids (primarily phosphatidyl choline, phosphatidyl ethanolamine and phosphatidyl serine) or to an increase in degra- dation products, such as free fatty acids and malonyldialdehyde. This effect has also been verified in the freezedried reagent [25], although the expiry periods are much longer.

After completing the first phase of the project in which samples were confined to small amounts sent only within the scope of the Basque Autonomous Community, and following recognition of our thromboplastin in 1998 as a standard for use in the Spanish External Quality Assessment Programme for Haematology, work will now begin on the process of freeze-drying.

\section{Acknowledgements}

This work was supported by grants No. 103.103-EA204/93, 103.103-EA131/95 and 103.103-EA211/96 from the University of the Basque Country (UPV/EHU), No. 0041-02/93 from the Spanish Health Ministry - Fondo de Investigaciones Sanitarias (FIS), and No. 1993-94, 1995-96 and 1996-97 from the Health Department of the Basque Country.

\section{References}

1 WHO Expert Committee on Biological Standardization: Guidelines for thromboplastins and plasma used to control oral anticoagulant therapy. World Health Organ Tech Rep Ser 1999;48.

2 Korsan-Bengtsen K, Johsen M, Pehrsson NG: Comparison between British Comparative Thromboplastin (BCT) and a factor II-VII-X determination method (Simplastin A) based on fresh samples from dicoumarol-treated patients. Thromb Haemost 1977;37:98-102.

3 Poller L, Thomson JM: National survey of value of plasma standards for anticoagulant therapy. Br Med J 1968;ii:88-92.

4 Poller L, Thomson JM: The Manchester Comparative Reagent. Boerhaave Series. Human Blood Coagulation. Leiden, University Press, 1969, pp 76-82.

5 Hirsh J, Poller L, Deykin D, et al: Optimal therapeutic range for oral anticoagulants. Chest 1989;95:5S-11S.

6 Poller L: The effect of the use of different tissue extracts on one-stage prothrombin times. Acta Haematol 1964;32:292-298.

7 lriarte JA: Terapéutica anticoagulante en nuestro medio. 10 años de experiencia en la FVPC. Med Clin (Barc) 1971;57:603-621.

8 Iriarte JA: Anticoagulant control. Lancet 1974; $\mathrm{i}: 730$

9 Iriarte JA: Control biológico de la anticoagulación. Jano 1976;28:55-59.

10 Poller L: A national standard for anticoagulant therapy. The Manchester Comparative Reagent. Lancet 1967;i:491-493.
11 Frei J, Hill PG, Heuck CC, El-Nageh MM, Riesen W, Poller L, Lang H: Production of Basic Diagnostic Laboratory Reagents. World Health Organisation Regional Publications, Eastern Mediterranean Series, 11. Alexandria, 1995.

12 Poller L: Prothrombin time (PT); in Jespersen J, Bertina RM, Haverkate F (eds): ECAT Assay Procedures, ed 2, rev. Laboratory Techniques in Thrombosis. A Manual. Dordrecht, Kluwer, 1999, pp 45-61.

13 van den Besselaar AMHP, Witteveen E, Shaefer-van Mansfeld H, Meeuwisse-Braun J, Strebus A: Accelerated degradation test of lyophilized recombinant tissue-factor liposome preparations. Thromb Haemost 1995;73:392-397.

14 WHO Expert Committee on Biological Standardization: Requirements for the collection, processing and quality control of blood components and plasma derivatives (requirements for biological substances No. 27, revised 1992). World Health Organ Tech Rep Ser 1994;43.

15 Martinez-Brotons F, Borrell M, Fontcuberta J, Batlle J, López F, Páramo JA, et al: Estudio multicéntrico comparativo entre una tromboplastina de conejo de alta sensibilidad y una tromboplastina recombinante con fosfolípidos sintéticos. Sangre 1994;39:245-251.

16 Peters RHM, van den Besselaar AMHP, Olthuis FMFG: A multicentre study to evaluate method dependency of the International Sensitivity Index (ISI) of bovine thromboplastin. Thromb Haemost 1989;61:166-169.
17 Tripodi A, Chantarangkul V, Braga M, Poller $\mathrm{L}$, ten Cate JW, van den Besselaar AMHP, et al: Results of a multicentre study assessing the status of a recombinant thromboplastin. Thromb Haemost 1994;72:261-267.

18 Hirsh J, Poller L, Deykin D, Levine M, Dalen JE: Optimal therapeutic range for oral anticoagulants. Chest 1989(suppl):5S-11S.

19 Poller L: Laboratory Control of Anticoagulant Therapy. Seminars in Thrombosis and Haemostasis 1986;12:13-19.

20 2nd ACCP Conference on Antithrombotic Therapy. Chest 1989;95:1S-169S.

21 Fourth ACCP Consensus Conference on Antithrombotic Therapy. Chest 1995;4:225S$306 \mathrm{~S}$.

22 Guidelines on Oral Anticoagulation, ed 3. Br J Haematol 1998;101:374-387.

23 Protocolos de Prevención y Tratamiento de la Enfermedad Tromboembólica. Madrid, Insalud, 1993.

24 Barcellona D, Biondi G, Vannini ML, Marongiu VF: Comparison between recombinant and rabbit thromboplastin in the management of patients on oral anticoagulant therapy. Thromb Haemost 1996;73:488-490.

25 Stevenson KJ, Slater PJ, Poller L: The British Comparative thromboplastin: The Relationship between Lipid Class Composition and Procoagulant Activity. Br J Haematol 1980;44: 495-501. 